\title{
Transcript profiling for early stages during embryo development in Scots pine
}

\author{
Irene Merino ${ }^{{ }^{*}}$, Malin Abrahamsson ${ }^{1}$, Lieven Sterck ${ }^{2,3,4}$, Blanca Craven-Bartle ${ }^{5}$, Francisco Canovas ${ }^{5}$ \\ and Sara von Arnold ${ }^{1}$
}

\begin{abstract}
Background: Characterization of the expression and function of genes regulating embryo development in conifers is interesting from an evolutionary point of view. However, our knowledge about the regulation of embryo development in conifers is limited. During early embryo development in Pinus species the proembyo goes through a cleavage process, named cleavage polyembryony, giving rise to four embryos. One of these embryos develops to a dominant embryo, which will develop further into a mature, cotyledonary embryo, while the other embryos, the subordinate embryos, are degraded. The main goal of this study has been to identify processes that might be important for regulating the cleavage process and for the development of a dominant embryo.

Results: RNA samples from embryos and megagametophytes at four early developmental stages during seed development in Pinus sylvestris were subjected to high-throughput sequencing. A total of 6.6 million raw reads was generated, resulting in 121,938 transcripts, out of which 36.106 contained ORFs. 18,638 transcripts were differentially expressed (DETs) in embryos and megagametophytes. GO enrichment analysis of transcripts up-regulated in embryos showed enrichment for different cellular processes, while those up-regulated in megagametophytes were enriched for accumulation of storage material and responses to stress. The highest number of DETs was detected during the initiation of the cleavage process. Transcripts related to embryogenic competence, cell wall modifications, cell division pattern, axis specification and response to hormones and stress were highly abundant and differentially expressed during early embryo development. The abundance of representative DETs was confirmed by qRT-PCR analyses.

Conclusion: Based on the processes identified in the $\mathrm{GO}$ enrichment analyses and the expression of the selected transcripts we suggest that (i) processes related to embryogenic competence and cell wall loosening are involved in activating the cleavage process; (ii) apical-basal polarization is strictly regulated in dominant embryos but not in the subordinate embryos; (iii) the transition from the morphogenic phase to the maturation phase is not completed in subordinate embryos. This is the first genome-wide transcript expression profiling of the earliest stages during embryo development in a Pinus species. Our results can serve as a framework for future studies to reveal the functions of identified genes.
\end{abstract}

Keywords: Embryo development, Pinus sylvestris L, Polyembryony, RNA-seq, Transcriptomic analysis, Zygotic embryogenesis

\footnotetext{
* Correspondence: irene.merino@slu.se

'Department of Plant Biology, Uppsala BioCenter, Swedish University of

Agricultural Sciences, Box 708075007 Uppsala, Sweden

Full list of author information is available at the end of the article
}

(c) The Author(s). 2016 Open Access This article is distributed under the terms of the Creative Commons Attribution 4.0 International License (http://creativecommons.org/licenses/by/4.0/), which permits unrestricted use, distribution, and reproduction in any medium, provided you give appropriate credit to the original author(s) and the source, provide a link to the Creative Commons license, and indicate if changes were made. The Creative Commons Public Domain Dedication waiver (http://creativecommons.org/publicdomain/zero/1.0/) applies to the data made available in this article, unless otherwise stated. 


\section{Background}

Post-embryonic development in plants depends on the establishment of stem cell niches in shoot and root meristems that take place during embryogenesis. Pattern formation in the embryo is under the control of coordinated spatially and temporally regulated gene expression, cell division, and hormone function. Most of our knowledge about the regulation of pattern formation during embryo development is based on studies of embryo-defective mutants in the angiosperm model plant Arabidopsis (Arabidopsis thaliana) [1]. By contrast, our knowledge about the regulation of embryo development in gymnosperms is limited. Molecular data suggest that extant seed plants (gymnosperms and angiosperms) shared a final common ancestor about 300 million years ago [2]. Therefore, characterization of the expression and functions of genes regulating embryo development in gymnosperms is interesting from an evolutionary point of view. Another reason to study gymnosperms, and especially conifers, is that they are of great commercial importance.

Embryo development in Pinus can be divided into three phases [3]: (1) proembryogeny - all stages before elongation of the suspensor, (2) early embryogeny - all stages during and after elongation of the suspensor and before establishment of the root meristem, (3) late embryogeny - establishment of the root and shoot meristem and further development of the embryo. Proembryogeny starts with a free nuclear stage. The zygote undergoes several rounds of nuclear duplication that are not followed by cytokinesis. After cell wall formation, four tiers are formed of which the lowest tier will form the embryonal mass and the second lowest tier will elongate to form the embryonal suspensor. In most Pinus species the four apical cells and the suspensor network in the proembryo separate into four filamentous embryos $[4,5]$. This process is termed cleavage polyembryony [6]. The four embryos, which arise from the separated tiers, begin their development by apical cell growth [7]. The basal cells of the embryonal mass divide anticlinally and elongate, contributing to the suspensor, which consists of several files of terminally differentiated non-dividing cells. Early embryogeny begins with the elongation of the suspensor. The enlarging suspensor pushes the embryo out of its archegonial jacket into the rich nutritive reserves of the megagametophyte. In an ovule where polyembryony is present, the competition between genetically identical embryos offers no selective advantage; instead it has been suggested that the embryo with the best physiological constitution situated in the most suitable environment becomes the dominant embryo, which usually develops to maturity [8]. The rest of the embryos, the subordinate embryos, are degraded by programmed cell death (PCD) [9]. During late embryogeny the root and shoot apical meristems are delineated and the plant axis established.
The maturing embryo is characterized by the initiation of cotyledons.

Various approaches have been taken for elucidating the regulation of embryo development in plants. The most comprehensive study of transcript profiling in a conifer was conducted in loblolly pine (Pinus taeda) where approximately 68,700 ESTs were regenerated from zygotic and somatic embryos [10]. Based on 295 genes, essential for embryogenesis in Arabidopsis, 85\% had very strong sequence similarity to an EST in the loblolly pine database [11]. Stress-related processes and auxinmediated-processes were, by using microarray analysis, identified to be associated with early somatic embryo development in Norway spruce [12]. Microarray analysis has also been performed for studying global gene expression during development of dominant zygotic embryos of maritime pine (Pinus pinaster) [13]. The results revealed that epigenetic regulation and transcriptional control related to auxin transport and response are critical during early to mid-stages of pine embryogenesis, and that important events during embryogenesis seem to be coordinated by putative orthologs of major developmental regulators in angiosperms. Recent advances in high-throughput sequencing technologies enable global transcriptome profiling without prior sequence knowledge [14]. By analysing the transcription network between embryo and endosperm during early seed development in maize (Zea mays) it was shown that many metabolic activities are specific for the embryo or the endosperm, and that transcription factors and imprinting genes are specifically expressed in the embryo or the endosperm [15]. Comparative transcriptome analysis of somatic and zygotic embryos in cotton (Gossypium hirsutum) uncovered that the process of somatic embryogenesis is characterized by induction of several stress-related genes [16]. Whole transcriptome profiling during initiation of embryogenic tissue in maize showed an increased expression of stress factors and the importance of a coordinated expression of somatic embryogenesis-related genes [17], as well as the involvement of a complex auxin-signalling pathway [18]. Several metabolic events were detected by transcriptome analysis in proliferating embryogenic cultures of Japanese larch (Larix leptolepis) [19].

To improve the understanding of genomic factors involved in early embryo development in Scots pine we performed a genome-wide high-throughput transcriptome sequencing for early stages during zygotic embryogenesis. The expression of twenty-three differentially expressed genes was confirmed by qRT-PCR analyses. Based on these analyses, and on the assumption that the Scots pine genes are homologous to their Arabidopsis counterparts, we have identified transcripts and putative processes that take place during early embryo development including initiation of cleavage polyembryogeny and development of 
dominant embryos. To our knowledge, this represents the first genome-wide transcript expression profiling of the earliest stages during embryo development in a Pinus species.

\section{Methods}

\section{Plant material}

Immature cones were collected for sequencing during summer 2012, from an open-pollinated seed orchard clone (W4009) of Scots pine (Pinus sylvestris L.) in Hade, central Sweden ( $60.3^{\circ}$ latitude, $17.0^{\circ}$ longitude). The Swedish Forestry Research Institute, that is running the seed orchard, had given us permission to collect cones. The same clone has been used for sequencing the Scots pine genome (WP1 in the European Community's Seventh Framework Programme, ProCoGen project). In order to collect the desired developmental stages of zygotic embryos and megagametophytes, cones were harvested periodically between the $11^{\text {th }}$ and $20^{\text {th }}$ of July. The zygotic embryos were excised from the megagametophytes under a stereomicroscope. Both the embryos (E) and the megagametophytes (M) were sorted and collected, in Eppendorf tubes placed on ice, according to developmental stage (Fig. 1): Stage 1, the ovules contained a single embryo at the stage before cleavage (E1, M1); Stage 2, the ovules contained an embryo at the stage of cleavage (E2, M2); Stage 3, the ovules contained a dominant embryo, DO, and subordinate embryos, SU (the dominant and the subordinate embryos were sampled separately, E3DO, E3SU, M3); Stage 4, the ovule contained a dominant embryo just before cotyledon differentiation (E4, M4). After a maximum of $10 \mathrm{~min}$ on ice, the Eppendorf tubes with collected embryos or megagametophytes were frozen in liquid nitrogen. Each sample included from 27 to 50 embryos, depending on the developmental stage. Equivalent materials were collected for qRT-PCR analyses during summer 2014. To avoid specificity of the embryogenesis process in a particular region, the new samples were collected from a tree of Scots pine growing at the SLU estate located in Ultuna, central Sweden (59.8 latitude, $17.7^{\circ}$ longitude), from which an unlimited number of cones could be collected. The harvesting of cones was performed periodically between the $17^{\text {th }}$ of June and $8^{\text {th }}$ of July.

\section{RNA extraction and CDNA synthesis}

Total RNA from zygotic embryos was extracted using the RNAqueous-Micro RNA Isolation Kit (Ambion), followed by a DNase I treatment to remove any residual genomic DNA, according to manufacturer's instructions. RNA from megagametophytes was isolated using the Spectrum Plant Total RNA kit (Sigma-Aldrich), including the On-Column DNAse I Digestion step for removing traces of genomic DNA.

The concentration of the isolated RNA from samples collected for RNA sequencing (one biological replicate) was determined fluorometrically using a Qubit fluorometer (Invitrogen), and the integrity was verified by an Agilent BioAnalyzer using the RNA 6000 Nano chip (Agilent Tecnologies). The RNA samples with an RNA integrity number (RIN) higher than 7 were used for cDNA synthesis and amplification with the Mint-2 cDNA synthesis kit (Evrogen). Briefly, first-strand synthesis was initiated from $1 \mu \mathrm{g}$ of total RNA by a Mint RT using a modified poly-dT primer. Second strand synthesis was carried out by the Encyclo DNA polymerase (Evrogen), followed by PCR amplification. The number of cycles (18 or 21) for doublestrand cDNA (dsDNA) amplification was estimated for each sample. Amplified cDNA was then purified with the NucleoSpin Gel and PCR Clean-up kit (Macheray-Nagel). Finally, a reamplification step was performed with specific primers for 454 pyrosequencing. In total, nine different dsDNA enriched libraries were constructed, five from zygotic embryos at stage E1, E2, E3DO, E3SU and E4, and four from megagametophytes at stage M1, M2, M3 and M4.

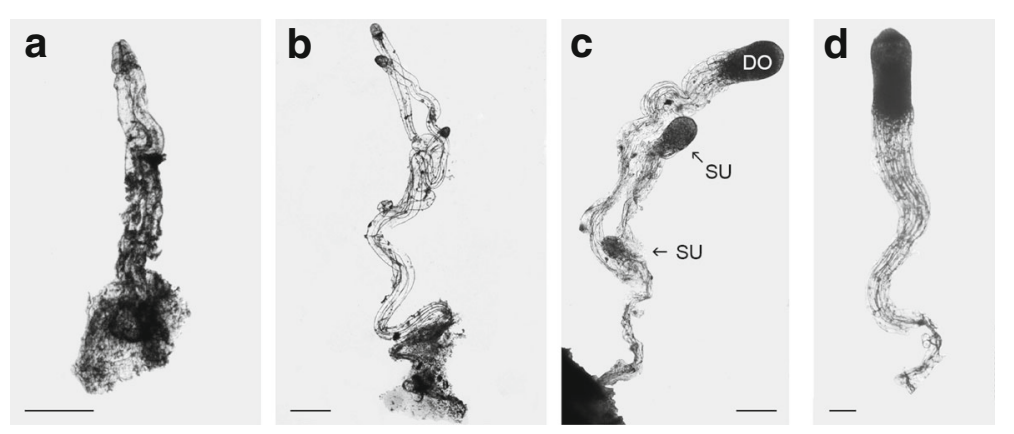

Fig. 1 Early stages during development of zygotic embryos in Scots pine that have been included in this study. a A single zygote-derived early embryo (stage 1; E1). b The single embryo at stage E1 has cleaved to form multiple embryos of equal size (stage 2; E2). c One embryo has become dominant (stage 3; E3DO) and subordinate embryos (stage 3; E3SU) successively stop developing. d A well-developed dominant embryo before cotyledon differentiation (stage 4; E4). Bars $0.5 \mathrm{~mm}$ 
RNA isolated from embryos collected for qRT-PCR (four biological replicates) was quantified using a NanoDrop1000 spectrophotometer (Nanodrop Technologies). cDNA synthesis from $100 \mathrm{ng}$ of total RNA was performed using the QuantiTect Whole Transcriptome (Qiagen) followed by $8 \mathrm{~h}$ amplification according to the manufacturer's protocol for high-yield reactions.

\section{Transcriptome sequencing}

Transcriptome sequencing was performed at the University of Malaga ultrasequencing facility using the GS-FLX + platform with a GS-FLX Titanium kit, Roche Applied Sciences (Indianapolis, IN, USA) following the protocol described by Canas et al. [20].

\section{Transcript reconstruction from RNA-seq}

Before assembly, the 6.6 million raw 454 reads were quality checked, and short reads ( $<75 \mathrm{bp})$ and adapter sequences were removed from the dataset using seqclean (Additional file 1: Table S1). After cleaning the reads were de novo assembled using the Newbler software (v2.8.1) which resulted in 76,425 isogroups containing 117,551 isotigs (Additional file 1: Table S2). In order to get an even more comprehensive transcriptome dataset, we then also incorporated publically available datasets with the previously obtained assembly. We integrated another 67,744 PUTs from PlantGDB (Resources for Comparative Plant Genomes) [21] and a set of 2161 ESTs from the NCBI ESTdb, which were used as a reference to map reads against (Additional file 1: Table S3). The various datasets were integrated using the CD-HIT software [22] in order to remove redundant transcripts and clustering into isogroups. For each isogroup only the longest isotig was retained for further analysis. This resulted in a final transcriptome set of 121,938 transcripts (Additional file 1: Table S3). The lengths of the assembled transcripts are shown in Additional file 1: Table S4.

Note: PUTs and $\mathrm{Cl} / 118$ transcripts that were not present in the seed transcriptome did not receive any reads, so their RPKM was 0 for all the stages and were then removed from the differential expression analysis.

For expression quantification of each sample, all cleaned reads were mapped back to the integrated transcript set using BWA [23]. Afterwards the mapping results were processed with samtools [24] to obtain read counts, which were then processed with an in-house PERL script to result in RPKM values for each transcript.

Open Reading Frame (ORF) predictions on the total 121,938 transcripts were obtained by applying TransDecoder with default parameters except for the coding model, which was specific for $P$. sylvestris, built from manually curated full length transcripts. TransDecoder identified 36,106 ORFs in the dataset.

\section{Functional annotation and enrichment analysis}

In order to functionally characterise the resulting ORFs, a blastP analysis (e-value cut-off 1e-5) was performed against the Arabidopsis TAIR10 database. All ORFs were also analysed for protein domains with interproscan (v5.13.52) [25] and possible GO-terms were determined based on the InterPro domains. To identify putative transcription factors (TFs) in our dataset all ORFs were screened against the Plant Transcription Factor Database, PlantTFDB v3.0 [26] using blastP (e-value cut-off 1e-5).

Gene annotation analyses and functional enrichment of differentially expressed transcripts in embryos and megagametophytes were performed with WeGO (Web Gene Ontology Annotation Plot) tool [27] and AgriGO analysis toolkit [28] respectively. For functional enrichment analyses the seed annotated transcriptome was used as background/reference genome. Hypergeometric test was used as statistical method with an adjusted FDR value (cut-off 0.05 ) and complete GO was selected as gene ontology type in the settings.

\section{Identification of differentially expressed transcripts}

For identification of differentially expressed transcripts (DETs), pairwise comparisons were performed between: (i) embryos and megagametophytes at the same developmental stage (E1 vs M1, E2 vs M2, E3DO vs M3, E3SU vs M3 and E4 vs M4), (ii) embryos at consecutive developmental stages (E1 vs E2, E2 vs E3DO, E3DO vs E4, E3DO vs E3SU) and (iii) megagametophytes at consecutive developmental stages (M1 vs M2, M2 vs M3, M3 vs $\mathrm{M} 4)$. The relative fold-change (FC) is presented as $\log 2$ of the RPKM ratio (sample A/sample B). Transcripts with a FC higher than 2 were considered as differentially expressed transcripts (DETs). When the RPKM value of one sample was 0 (no expression detected) the foldchange could not be estimated. In these cases 99 and -99 values were assigned as relative fold-changes. In addition, when the RPKM value was 0 in one of the samples and lower than 10 in the other, the transcript was excluded from differential expression analyses.

Venn diagrams have been drawn with the online web tool available at http://bioinformatics.psb.ugent.be/webtools/Venn/.

K-means cluster analysis was performed with a subset of DETs (FC higher than 2 and RPKM over 10) detected in any of the pairwise comparisons between different developmental stages in embryos and in megagametophytes. Initially, the relative expression value for each DET was calculated by normalizing all the RPKM values from different developmental stages to its maximum RPKM value. The optimal number of clusters was estimated separately for the embryo and megagametophyte data and normalized values were clustered using the kmeans function in $\mathrm{R}$ software. 


\section{Quantitative RT-PCR}

Quantitative RT-PCR was performed in a Bio-Rad CFX Connect $^{\mathrm{TM}}$ Real-Time PCR Detection System cycler (BioRad Laboratories). All samples were run in duplicate starting from $5 \mathrm{ng}$ of cDNA from four biological replicates for each developmental stage. ELONGATION FACTOR 1 (EF1) and PHOSPHOGLUCOMUTASE (PHOS) were used as reference genes [12]. Relative quantitative analyses were performed following the $2^{-\Delta \Delta \mathrm{Ct}}$ Livak method. Only transcripts showing a similar expression profile in at least three out of four biological replicates have been included. The primer sequences for the transcripts tested are shown in Additional file 2: Table S5. Significant differences in transcript accumulation between different developmental stages were estimated by a $t$-test mean comparison analysis $(P \leq 0.05)$ using the JMP software (v11).

To validate the RNA-seq data, the same RNA that was used for sequencing was tested by qRT-PCR. New cDNA from embryos was synthesized and amplified using the QuantiTect Whole Transcriptome kit (Qiagen), as has been explained above. cDNA from megagametophytes was synthesized by using the PrimeScript ${ }^{\mathrm{TM}} \mathrm{RT}$ reagent Kit (Takara), according to manufacturer's instructions. The Pearson correlation coefficient between the expression profiles obtained by RNA-seq and qRT-PCR was calculated for each of the 23 candidate transcripts in embryos and for 7 selected DETs (involved in response to stress and stimulus) in megagametophytes (Additional file 2: Table S6). The correlation coefficient was estimated by using the Pearson statistical function in Microsoft Excel.

\section{Results and discussion}

\section{Overview of the transcriptome in seeds}

To identify transcripts and biological processes involved in early zygotic embryogenesis in Scots pine, RNA was isolated from embryos and megagametophytes representing four developmental stages (Fig. 1). Nine RNAseq libraries were sequenced by using 454 Roche sequencing technology. A total of 6.6 million raw reads was generated, resulting in 121,938 transcripts varying in length from 150 to $18,101 \mathrm{bp}$ and with a mean length of 1242 bp (Additional file 1: Tables S1, S2, S3 and S4).

In total, 36,106 transcripts containing ORFs were identified in the seed transcriptome, of which 28,190 transcripts (78\%) had significant alignments to the Arabidopsis thaliana TAIR10 database and 7404 transcripts (20\%) with the Plant Transcription Factor Database (Table 1). 26,743 transcripts (74\%) had annotated GO terms into at least one of the three main categories: 22,362 transcripts (60\%) displayed one or more ontologies related to Biological Process, 24,259 (67\%) to Molecular Function and 19,301 (53\%) to Cell Component.
Table 1 Summary of RNA-seq seed transcriptome data

\begin{tabular}{llll}
\hline & All samples & $\begin{array}{l}\text { Embryo } \\
\text { samples }\end{array}$ & $\begin{array}{l}\text { Megagametophyte } \\
\text { samples }\end{array}$ \\
\hline Transcripts with RPKM >0 & 81,120 & 74,149 & 59,524 \\
Transcription factors & 7404 & 7200 & 6605 \\
Transcripts with ORFs & 36,106 & 29,595 & 26,400 \\
Transcripts with hits against & 28,190 & 24,043 & 22,556 \\
TAIR database & & & \\
Annotated transcripts (GO) & 26,743 & 25,441 & 23,309 \\
\hline
\end{tabular}

Transcript expression values were calculated as RPKM, resulting in 81,120 assembled transcripts with detectable expression signals (RPKM $>0$ ), in at least one of the developmental stage (Table 1). 74,150 and 59,526 transcripts were detected in embryos and megagametophytes, respectively. Most of the transcripts (65\%) were detected in both tissues, however the number of unique transcripts was threefold higher in embryos than in megagametophytes (Fig. 2a). The number of identified transcription factors (TFs) was also higher in embryos (Fig. 2b).

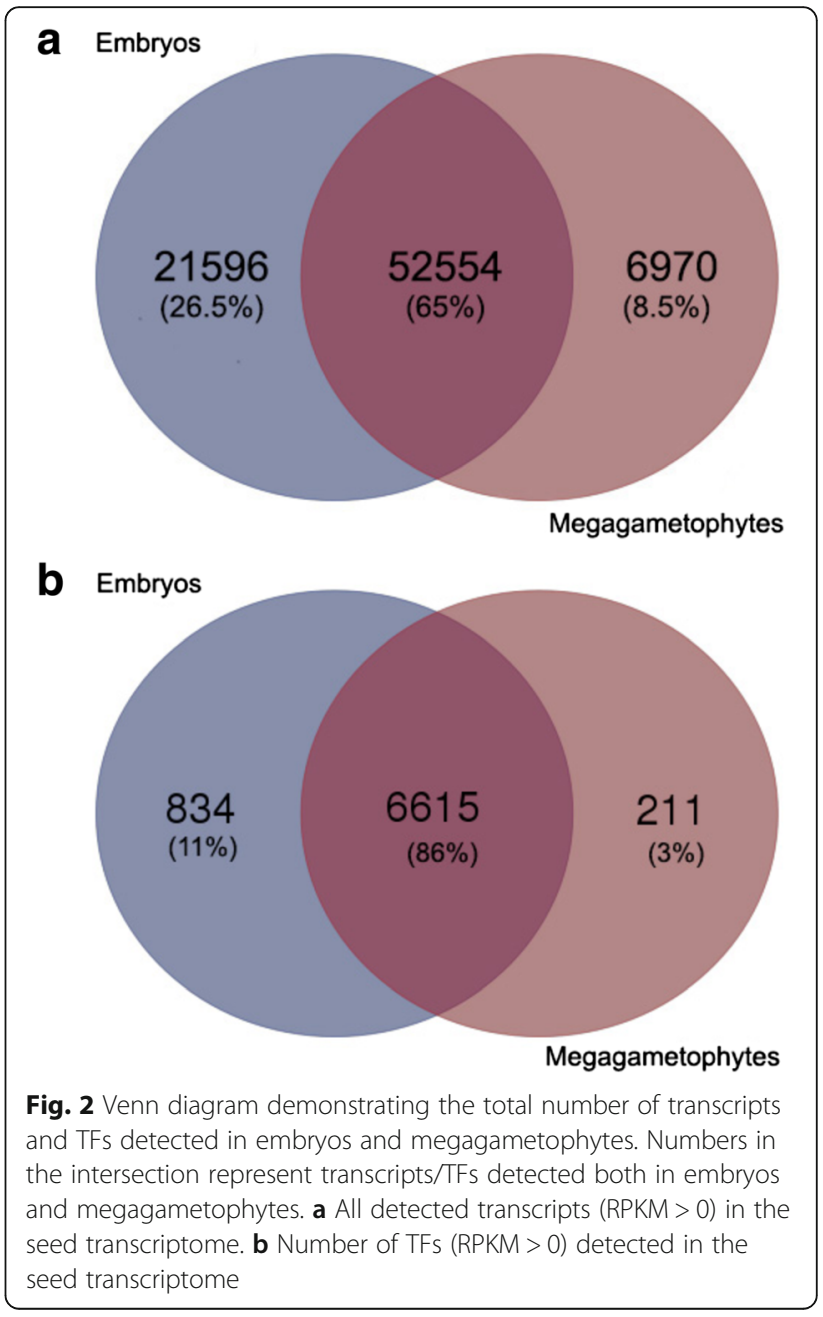


The total number of transcripts detected at each developmental stage during seed development increased in embryos, but decreased in megagametophytes (Table 2). Around 15,000 transcripts were expressed at all developmental stages both in embryos and in megagametophytes. The number of unique transcripts detected at specific developmental stages was fairly constant in the embryos, but decreased in the megagametophytes during seed development from 10,907 transcripts at stage M1 to 3201 transcripts at stage M4 (Additional file 1: Figure S1A and B). Out of 7404 TFs identified during early embryo development, 3734 TFs (50\%) were detected at all developmental stages, and about 140 TFs were only detected at one developmental stage (Additional file 1: Figure S1C). In megagametophytes, 3775 TFs (56\%) were detected at all developmental stages, however, the number of TFs detected at only one developmental stage decreased during seed development (Additional file 1: Figure S1D).

To test the reliability of the RNA-seq results, 30 transcripts (23 transcripts in embryos and 7 transcripts in megagametophytes) were selected for examination by qRTPCR. The Pearson correlation coefficient between the expression profiles obtained by RNA-seq and qRT-PCR was calculated from each transcript separately (Additional file 2: Table S6). The correlation coefficient obtained was similar for most transcripts, except for a few transcripts at some time points.

\section{Changes in transcript accumulation during seed development}

Differentially expressed transcripts in pairwise comparisons between embryos and megagametophytes during seed development

To identify differentially expressed transcripts (DETs) we performed pairwise comparisons between embryos and megagametophytes at the same developmental stage. In total 18,638 transcripts were up-regulated with a fold change higher than $2(\mathrm{FC}>2)$ in at least one of the pairwise comparisons between embryos and megagametophytes (Additional file 3: Figure S2A, Additional file 4). 12,906 transcripts were up-regulated in embryos and 5732 in megagametophytes. The greatest difference in the number of up-regulated transcripts between embryos

Table 2 Number of transcripts and TFs detected in embryos and megagametophytes at different developmental stages (RPKM >0)

\begin{tabular}{llllll}
\hline Embryos & E1 & E2 & E3DO & E3RE & E4 \\
\hline Transcripts & 39,423 & 43,309 & 46,976 & 46,492 & 47,089 \\
TFs & 4993 & 5725 & 5936 & 5641 & 5810 \\
Megagametophytes & M1 & M2 & M3 & & M4 \\
Transcripts & 40,595 & 38,853 & 35,188 & & 34,235 \\
TFs & 5468 & 5249 & 5073 & & 4989 \\
\hline
\end{tabular}

and megagametophytes was observed at developmental stage 2 (Additional file 3: Figure S2B).

About $54 \%$ of the DETs up-regulated in embryos and $58 \%$ of the DETs up-regulated in megagametophytes could be GO annotated (Additional file 3: Figure S2A). Cellular and metabolic processes were the most dominant groups in the Biological Process category both in embryos and megagametophytes (Fig. 3). Furthermore, transcripts assigned to response to stimulus were overrepresented in megagametophytes. In both embryos and megagametophytes, enriched GO terms in the Molecular Function category included catalytic and binding activities, and in the Cell Component category the subcategories cell and cell part were the most abundant.

By increasing the GO annotation level, it was found that transcripts up-regulated in embryos were enriched for diverse Biological Processes such as cellular component biogenesis and cellular and metabolic processes related to chromosome organization, DNA packaging, translation and gene expression (Fig. 4a and Additional file 3: Figure S3). In the megagametophytes the up-regulated transcripts were highly enriched in response to stimulus, such as response to stress and to chemical and endogenous stimulus, including response to abscisic acid (ABA) (Fig. 4b and Additional file 3: Figure S4). In the Molecular Function category, assignments in the embryos were mainly related to DNA binding and structural constituent of ribosome. Both activities are highly related to gene expression and protein synthesis. In the megagametophytes, transcripts functioning in nutrient reservoir activity were highly over-represented $($ FDR $=2.82 \mathrm{e}-92)$. Transcripts identified in embryos for the Cell Component category showed enrichment for nucleus, ribosome and protein-DNA complex (Fig. 4a) and transcripts in megagametophytes were enriched mainly in protein body component (Fig. 4b). As expected, transcripts up-regulated in embryos showed GO enrichment for different cellular processes and functions in DNA-packaging, translation and gene expression. These processes are important during active cell proliferation [29]. Transcripts up-regulated in megagametophytes were enriched for accumulation of storage material and response to chemical and endogenous stimuli. This might indicate that the megagametophyte, in a similar way as the endosperm, can sense environmental signals and induce the corresponding signalling pathways for regulating embryo development [30].

We carried out pairwise comparisons between the group of transcripts showing the highest differences in abundance between embryos and megagametophytes at each developmental stage (Additional file 5). Transcripts related to members of the Arabidopsis cytochrome P450 gene family (CYP78A7, CYP78A8 and CYP71B22) showed, at all developmental stages, high accumulation in embryos but low or no accumulation (RPKM close to 0) in megagametophytes. Up-regulated transcripts in megagametophytes were mainly 


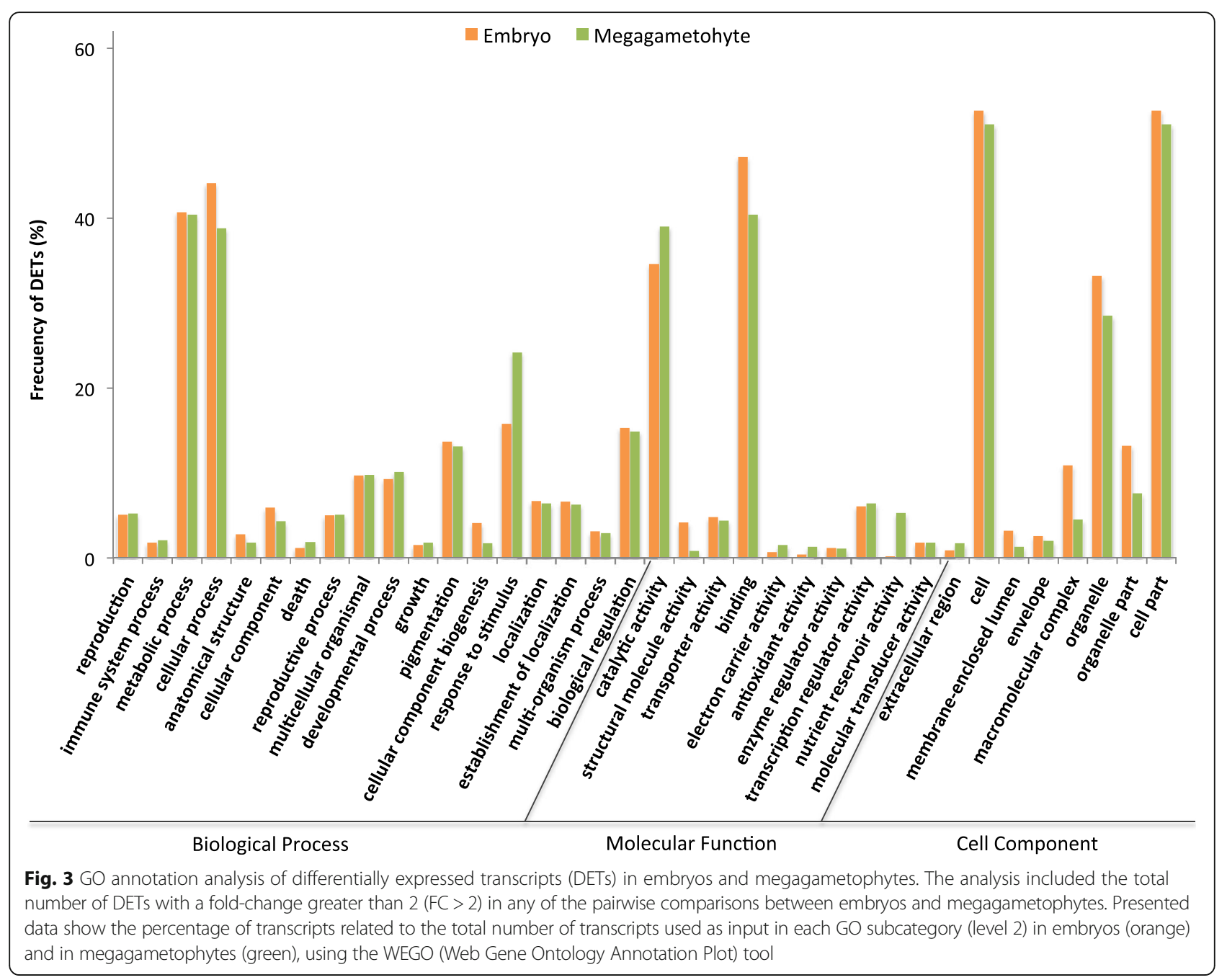

related to the Arabidopsis $12 \mathrm{~S}$ seed storage protein family $(C R B, C R C, C R D)$, also known as cruciferins. These proteins are involved in nutrient reservoir activity and are the major sources of nitrogen and carbon during early seed germination [31]. The RPKM values of cruciferin-related transcripts were similar at all developmental stages. The majority of the transcripts up-regulated in the megagametophytes had no hits against the TAIR database (Additional file 5).

At stage E1, E2 and E3DO, transcripts related to genes encoding for cell wall modifications (expansins, cellulose metabolism, endoglucanase, pectin-acetylesterase and pectinlyase) were detected. Specifically at stage E1, a putative homolog to SOMATIC EMBRYOGENESIS RECEPTORLIKE KINASE1 (SERK1), as well as transcripts related to genes involved in response to auxin and other hormones such as INDOLE-3ACETATE O-METHYLTRANSFERASE 1 (IAMT1), SKP1-LIKE PROTEIN 1A (SKP1A), GAMMAVACUOLAR-PROCESSING ENZYME (GAMMA-VPE), GIBBERELLIN-REGULATED PROTEIN 2 (GASA2) and
GLUTATHIONE S-TRANSFERASE U17 (GSTU17) were highly abundant (Additional file 5, Up in E1). Transcripts related to nucleosome assembly (histones) were detected at all developmental stages except at stage E1. Other transcripts up-regulated from stage 2 onwards coded for proteins related to stress responses i.e. non-specific LIPID-TRANSFER PROTEIN 3 (LTP3), SUGAR TRANSPORT PROTEIN 13 (STP13) or ABSCISIC ACID INSENSITIVE 4 (ABI4). Transcripts up-regulated at stage E4 included transcripts related to cell signalling, negative regulation of cell division and cell wall loosening, as well as transcripts related to development such as PROTEIN RALF-like 34 (RALFL34), FAMA, PLANTACYANIN (ARPN) or PECTIN ACETYLESTERASE (PAE9) (Additional file 5, Up in E4).

In total 7704. TFs were detected during early seed development (Table 1). Out of these TFs, 2890 were differentially expressed with a fold change higher than two between embryos and megagametophytes (Additional file 6). The differentially expressed TFs belonged to 78 families, of which the bHLH, FAR1, TRAF and NAC families were the largest 


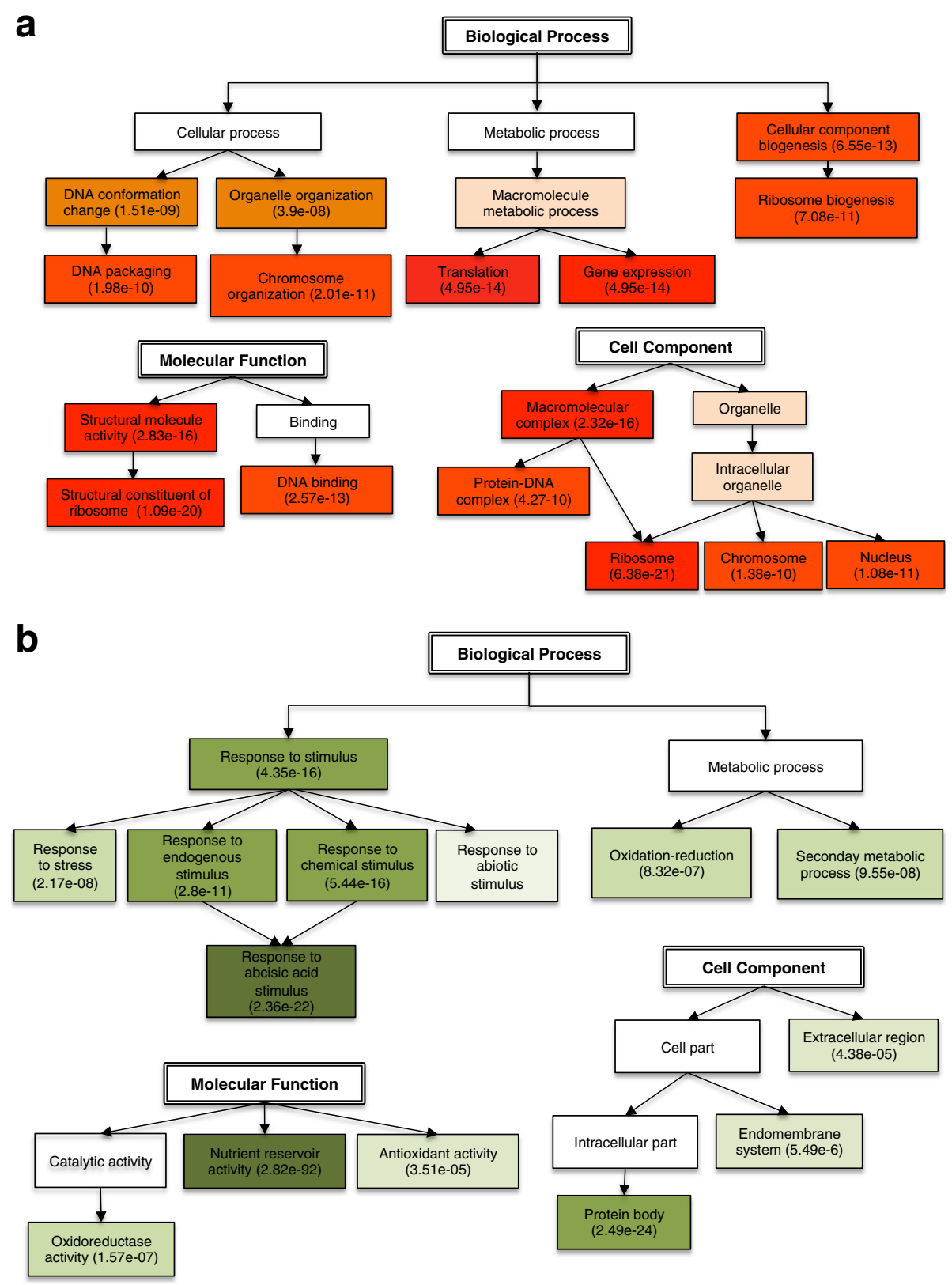

Fig. 4 Summary of Gene Ontology (GO) enrichment analysis for differentially expressed transcripts during early seed development. The analysis included DETs with a fold change greater than 2 ( $F C>2$ ) identified in any of the pairwise comparisons between embryos and megagametophytes. The most abundant classes in each category, Biological Process, Molecular Function and Cell Component are shown for $\mathbf{a}$ embryos and $\mathbf{b}$ megagametophytes. Level of enrichment is proportional to color intensity. FDR values are presented in parenthesis. Detailed information is shown in Additional file 3: Figures S2 and S3

(Additional file 7: Figure S5 and Additional file 6, Family distribution). In general, the number of TFs belonging to each family was higher in embryos than in megagametophytes. Interestingly, some of the TF families were enriched differently in embryos and megagametophytes during seed development e.g. for bHLH, C3H, NAC, AP2-EREBP and TRAF (Additional file 7: Figure S6). In addition, sixteen TF families were detected only in embryos and four TF families were detected only in megagametophytes. In general, several TFs belonging to families specifically expressed in embryos were involved in plant growth and development, while TF families detected only in megagametophytes were related to responses to stress and other stimuli [32-34].

\section{Differentially expressed transcripts during embryo development}

In total, 18,234 DETs with a fold change higher than two were identified in the pairwise comparisons between 
embryos at different developmental stages (Additional file 8: Figure S7). When including only transcripts with a RPKM > 10, 6669 DETs were detected. To provide an overview of the expression patterns of these DETs during embryo development, k-means clustering analysis was performed (DETs from subordinate embryos were excluded from this analysis). Four types of expression profiles were detected, where type I and II included four clusters each and type III and IV include two clusters (Fig. 5). The accumulation of transcripts belonging to type I increased throughout the course of embryo development. Transcripts in cluster 1 and 8 were specifically enriched for processes related to response to abiotic stress, and transcripts in cluster 3 and 7 were highly enriched for nutrient reservoir activity $(\mathrm{FDR}=2.10 \mathrm{e}-47$ ), response to $\mathrm{ABA}$ and other hormones. The expression of type II transcripts decreased during embryo development. However, the accumulation pattern differed among the four clusters. Transcripts in cluster 9 and 12 were abundant for cell wall modification, toxin and carbohydrate metabolic processes, while cluster 11 included a higher number of transcripts with a function in structural constituents of ribosomes. Type III transcripts showed high accumulation at only one intermediate developmental stage (E2 or E3DO). Transcripts within cluster 5, mainly accumulated at stage E2, were highly enriched for nutrient reservoir activity. However, no significant GO enrichment was obtained for cluster 4 . The expression level of type IV transcripts was either high or low at both E2 and E3DO stages. Cluster 2 included transcripts involved in DNA packaging and proteinDNA complex assembly. Together the GO enrichment analyses of the clusters showed that the abundance of transcripts related to stress response and nutrient activity increased during embryo development, while the abundance of transcripts related to cell wall modification decreased.

When comparing embryos at consecutive developmental stages, including subordinate embryos, 4411 DETs were detected. The highest number of DETs (2667) was detected in the comparison between embryos at stage E1 and E2, and $80 \%$ (2152) of these DETs were only detected in this pairwise comparison (Fig. 6a and b). DETs highly accumulated at stage E1, were enriched for Biological Processes related to cell wall loosening, organization and modification, with a beta-expansin (EXPB1)-related transcript having the highest fold-change (Additional file 9, E1xE2 Up). Furthermore, 28 TFs involved in several developmental processes were detected, out of which transcripts related to $L O B$ DOMAINCONTAINING PROTEIN 29 (LBD29) and SERK1, as well as some members belonging to the homeobox-leucine zipper protein family (HAT5 and HB13), showed a high foldchange (Additional file 8: Table S7 and Additional file 10, E1xE2 Up). Transcripts that were over-represented in E2 were enriched for processes related to response to $A B A$, hormone stimulus, nucleosome organization and nutrient reservoir activity (Additional file 9, E1xE2 Down). These DETs included 21 TFs that were GO annotated for developmental processes (Additional file 8: Table S7 and Additional file 10, E1xE2 Down).

Close to 660 DETs were identified when comparing embryos at stage E2 and E3DO (Fig. 6a). Transcripts assigned to response to $\mathrm{ABA}$ and hormone stimulus showed higher accumulation at stage E2, and those involved in response to abiotic stress were enriched at stage E3DO (Additional file 9, E2xE3DO). When comparing dominant embryos at stage E3DO and stage E4, 1087 DETs were detected (Fig. 6a). Transcripts upregulated in E3DO embryos were mainly related to axis specification processes, while transcripts up-regulated at stage E4 were involved in processes related to response to hormone stimulus and lipid transport (LTP3 and LTP4) (Additional file 9, E3DOxE4 Down). TFs, differentially expressed in embryos at stage E3DO and E4, which were annotated to developmental processes, included transcripts related to AUXIN RESPONSIVE FACTOR 2 (ARF2), LEUNIG (LUG), WUSCHEL-RELATED HOMEOBOX (WOX), CYP78A7 and ARABIDOPSIS NAC DOMAIN CONTAINING PROTEIN 9 (ANACOO9) (Additional file 8: Table S7 and Additional file 10).

By comparing dominant and subordinate embryos at stage E3, it was possible to detect 748 DETs (Fig. 6a). Many of the transcripts up-regulated in dominant embryos were related to carbohydrate metabolic processes and axis specification processes (Additional file 9, E3DOxE3SU Up). DETs enriched in subordinate embryos were involved in response to water stress (including water deprivation) and lipid transport. NAC and $\mathrm{HB}$ were the largest TF families in dominant embryos, while in subordinates MYB-related factors were the most abundant (Additional file 10, TF families).

A schematic summary of the results obtained from the pairwise comparisons between consecutive stages during embryo development is presented in Fig. 7. Together our results show that processes involved in cell-wall modifications, hormone signalling, axis specification and stressinduced responses are activated during early embryo development. A strict regulation of cell division, elongation and adhesion is critical during embryonic patterning formation. Auxin is perhaps the most pervasive signalling molecule in plants and has been implicated in many developmental processes including embryogenesis in both angiosperms and conifers [35-37]. In several studies it has been shown that genes related to stress are over-represented during early embryo development [12, 16, 17, 38]. Furthermore, many of the differentially expressed TFs that belong to the largest families (bHLH, FAR1, NAC and AP2-EREBP) are related to cellular and developmental processes, hormone signalling and stress responses [39-42]. 


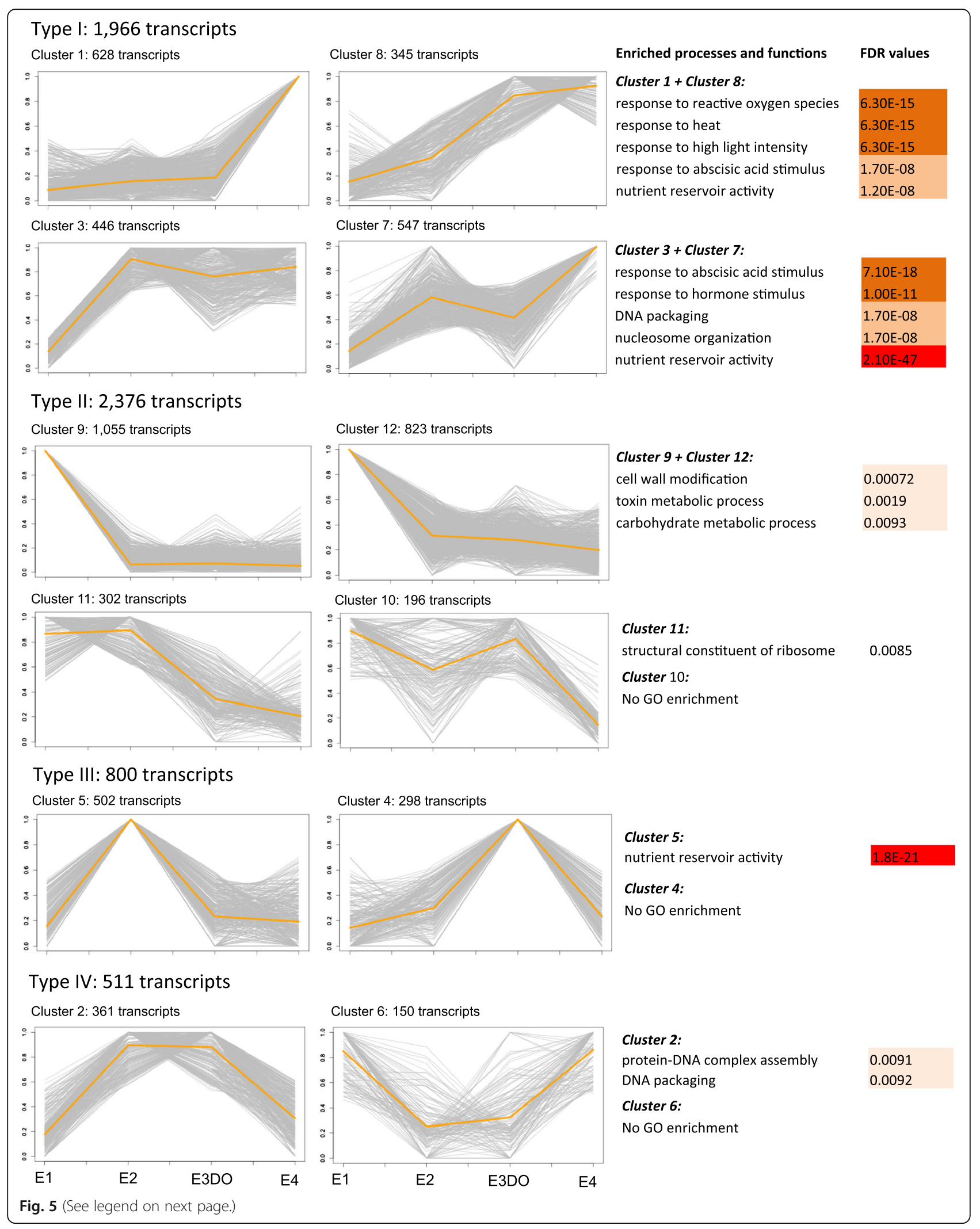


(See figure on previous page.)

Fig. 5 Clustering of differentially expressed transcripts identified in the pairwise comparisons between embryo developmental stages. The analysis included DETs with a FC $>2$ and RPKM > 10. For each transcript, RPKM values were normalized to its maximum RPKM value during embryo development. Normalized values were subjected to k-means clustering method and classified into 12 different clusters, based on their expression level across the four developmental stages. The Y-and X-axis represent relative expression (from 0 to 1) and different embryo developmental stages, respectively. Enriched processes and functions and their enrichment level (FDR) are presented for each cluster. The level of enrichment is proportional to colour intensity

\section{Differentially expressed transcripts between different developmental stages in megagametophytes}

DETs identified in the pairwise comparisons between megagametophytes at consecutive developmental stages were also subjected to $\mathrm{k}$-means clustering, resulting in 12 different clusters grouped into five types of expression profiles (Additional file 11: Figure S8). No significant

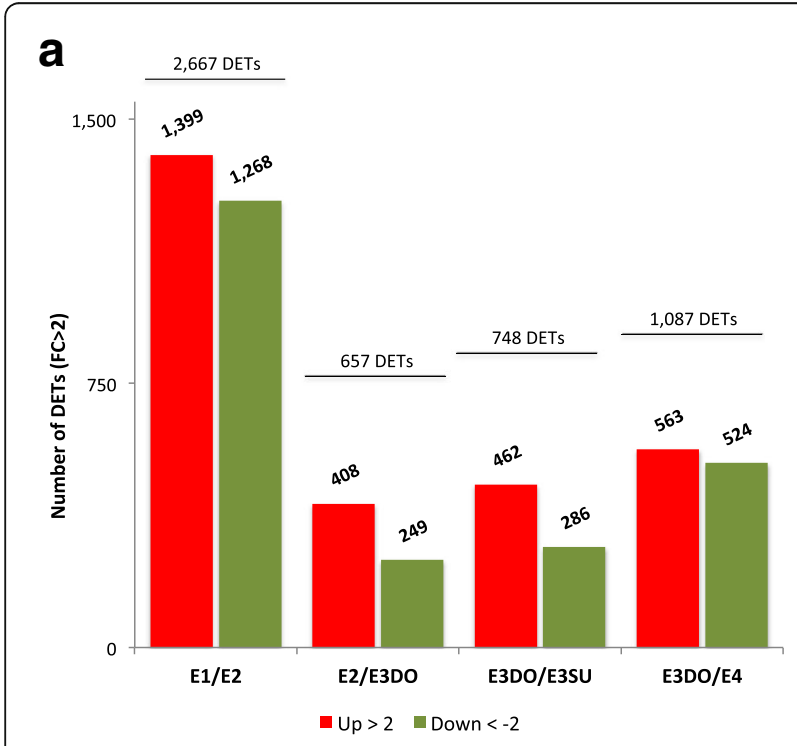

b

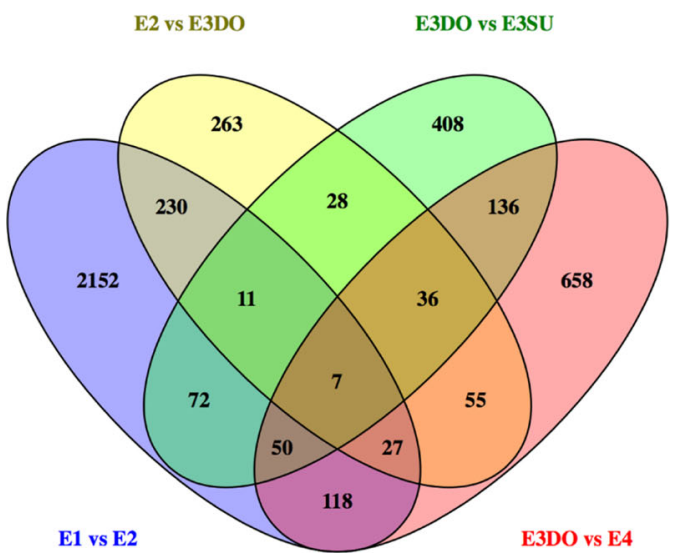

Fig. 6 Differentially expressed transcripts (DETs) between consecutive stages during embryo development. The analysis included DETs with FC $>2$ and RPKM $>10$ identified in any of the four pairwise comparisons. a Histogram showing the number of up-regulated (red bars) and downregulated (green bars) DETs between consecutive developmental stages. b Venn diagram showing the number of common and specific DETs between consecutive pairwise comparisons
GO enrichment processes or functions $(\mathrm{FDR}<0.05)$ were assigned to any of the clusters. The accumulation of type I transcripts increased from stage M1 to stage M4. Transcripts related to response to stimulus and regulation of biological process were the most abundant. The expression of type II transcripts decreased from stage M1 to stage M4. Type II clusters were abundant in transcripts with GO terms associated with cell wall organization, and reproductive and developmental processes. Type III transcripts accumulated either at stage M2 or stage M3. GO terms assigned to clusters in type III were mainly related to response to stimulus. Type IV and V included only one small cluster each. Cluster 6, with transcripts accumulating both at stages M2 and M3, presented a high percentage of DETs responding to stimulus, while transcripts from cluster 11 were annotated only for metabolic and cellular processes.

A total of 600 DETs $(\mathrm{FC}<2, \mathrm{RPKM}>10)$ were detected in the pairwise comparisons between megagametophytes at consecutive developmental stages. No significant enriched processes or functions were found in any of the pairwise comparisons. Similar to embryos, the highest number of DETs was detected during the transition from stage M1 to $\mathrm{M} 2$, and $85 \%$ of the DETs were specifically detected in this pairwise comparison (Additional file 11: Figure S9A and B). The number of transcripts annotated for developmental processes decreased from 10 at stage M1 to 2 at stage M4. Meanwhile, the number of DETs with GO terms associated with response to stress and stimulus remained more constant (Additional file 11: Figure S9C). In addition, transcripts encoding for proteins belonging to the small Heat Shock Protein (sHSP) family, known for its role in stress response, showed similar accumulation during all developmental stages (Additional file 12, M1xM2 Down, M2xM3 Up).

Ten DETs with assigned GO terms related to development were specifically detected at stage M1, in which putative homologs to expansins (EXPB1s) and $A G A$ MOUS-like MADS-box (AGL11) were included. AGL11 was not detected in embryos at any developmental stage. In accordance, $A G L 11$ was expressed in the endosperm but not embryos of Brachypodium distachyon (Expression Atlas data from EMBL-EBI). Several transcripts related to metal ion transport, e.g. Copper Transporter 5 (COPT5) and Zinc Transporter 11 (ZIP11), were detected at stage M1 (Additional file 12, M1xM2 Up). ZIP transporters participating in ion translocation during embryo and endosperm development have been detected 


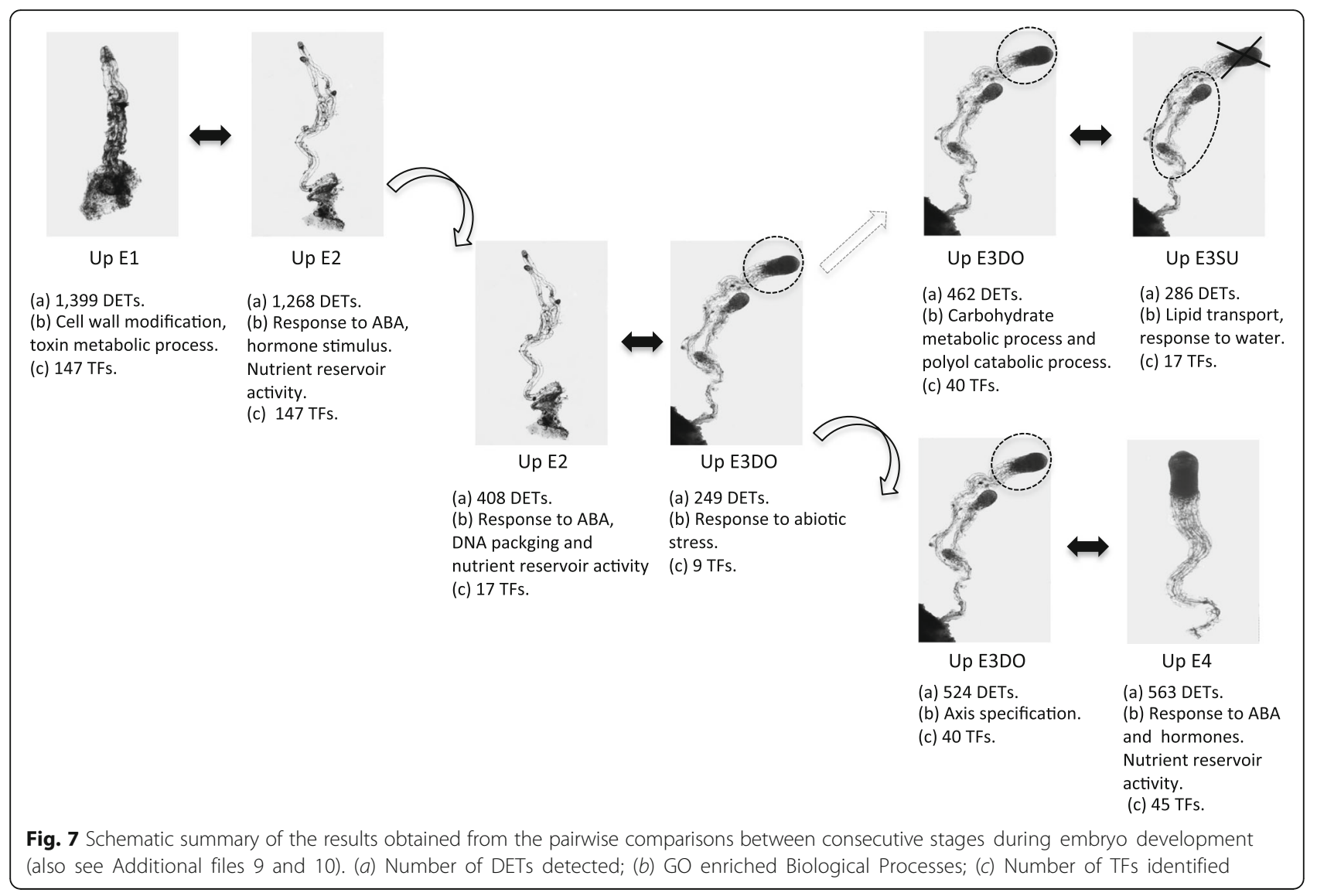

in maize seeds [43]. At stage M4, a transcript related to AtEP3, encoding for an endochitinase, was highly abundant (Additional file 12, M3xM4 Down). A homologous gene, Chia4-Pa1, has been shown to be expressed in the single cell-layered zone surrounding the corrosion cavity in the megagametophyte in Norway spruce seeds [44].

\section{Expression of selected transcripts during early embryo development}

The transcript levels of selected DETs were tested by qRT-PCR in four biological replicates. The selection was based on the estimated expression (RPKM values) obtained from the transcriptome data and functional annotations of homologous genes in other species, mainly Arabidopsis, that have been related to embryo development. Gene sequence information in conifers is limited, thus for convenience, we refer each conifer transcript to the Arabidopsis gene that it shares most sequence similarity to. We have taken this approach for making it possible to get a general idea about which processes might be important during early embryo development. The results generated from qRT-PCR analysis are presented in Fig. 8.

Transcripts related to ENDO-BETA-MANNASE 7 (MAN7), TRANSPARENT TESTA7 (TT7), EXPB1, SERK1,
LTP4, and HAP3A were highly abundant at stage E1 and decreased significantly at stage E2.

In Arabidopsis seeds, the mannanase-encoding gene, AtMAN7, is expressed in the micropylar endosperm and in the radicle tip just before radicle emergence [45]. We assume that the high expression of PSMAN7 in E1 embryos might facilitate their penetration into the nutritious megagametophyte.

Early embryogenesis is a critical developmental phase when the apical-basal polarity is established through directional auxin transport mainly mediated by auxin influx and efflux carriers [46]. In addition, flavonols can act as negative regulators of auxin transport [47]. AtTT7 encodes flavonoid 3'hydroxylase, a flavonol biosynthetic enzyme. Down-regulation of PSTT7 from stage E2 might reflect that auxin transport is increased from the cleavage stage and during further embryo development. Previous studies have associated the action of expansins in cell wall loosening, expansion, dissemble or separation [48, 49]. A high expression of PsEXPB1 at stage E1 might indicate the importance of loosening the cell walls to allow separation of the four early embryos. AtSERK1 marks cells that are competent to form embryos, and it also influences the competence of the cells to differentiate into embryos [50]. The high expression of PSSERK1 at stage E1 might be 


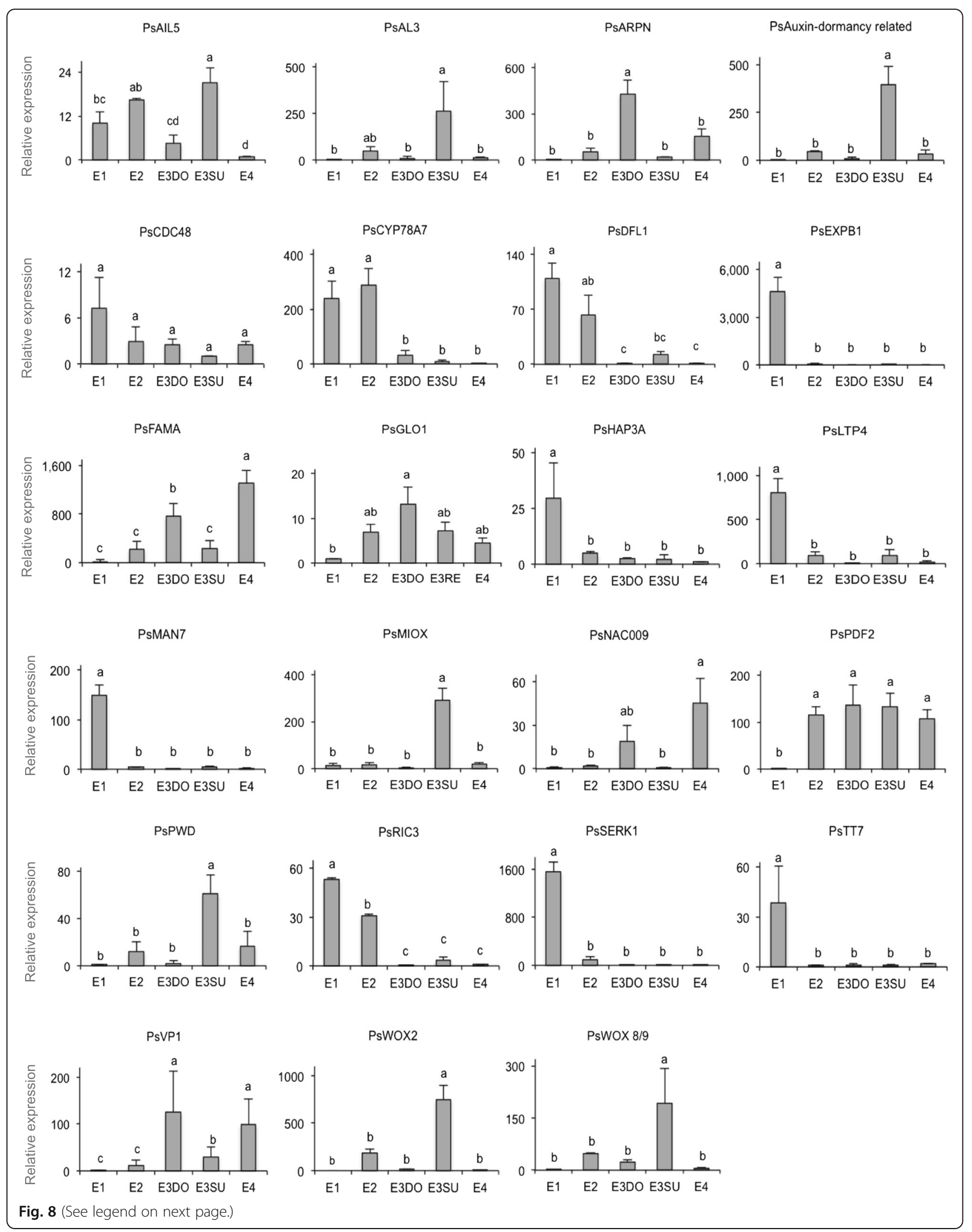


(See figure on previous page.)

Fig. 8 Quantitative real-time (qRT)-PCR analysis of the relative transcript level of selected genes during early embryo development. Relative expression levels, estimated by the Livak method (2- $\Delta \Delta \mathrm{Ct})$, are referred to the developmental stage showing the minimum accumulation for each transcript. Expression values are normalized against two reference genes ELONGATION FACTOR-1 (EF1) and PHOSHOGLUCOMUTASE (PHOS). Transcript levels are means \pm SD of three or four biological replicates, with two technical replicates each. Different letters indicate significant differences in the relative expression level (Student's t-test, $P<0.05$ )

important for stimulating the four apical cells to differentiate into separate embryos and thereby stimulate the cleavage process to start. Directly after the first cleavage, the four new embryos should develop further. We assume that down-regulation of PSSERK1 is important for blocking a second round of cleavage.

Another set of transcripts, represented by putative homologs to CYP78A7, DWARF IN LIGHT 1 (DFL1) and ROP-INTERACTIVE CRIB MOTIF-CONTAINING PROTEIN 3 (RIC3), were also highly abundant at stage E1 but declined successively during later developmental stages.

Cytochrome P450s are involved in the metabolism of most phytohormones and many secondary metabolites in plant cells. Overexpression of a member of the CYP78A family in rice (Oryza sativa) promotes cell proliferation but reduces the size of the embryos [51]. Furthermore, the gene product of AtDFL1, which is involved in auxin signal transduction, can inhibit cell elongation [52]. Before the development of the dominant embryo, the four early embryos are equal-sized. Although it is not known which mechanisms are restricting the growth of the embryonal mass of stage 2 embryos, our results indicate that PsCYP78A and PsDFL1 might be involved. AtRIC3 is important for tip growth of pollen tubes [53]. Early embryos in Pinus, developing after the cleavage process, begin their development by apical cell growth [7]. A high expression of PsRIC3 at stage E1 and E2 might reflect that these embryos develop by apical cell growth.

The level of transcripts related to NAC009, FAMA, PROTODERMAL FACTOR2 (PDF2), and VIVIPAROUS1 (VP1) were low at stage $\mathrm{E} 1$ but increased during later stages. $\mathrm{Pu}-$ tative homologs to PLANTACYANIN (ARPN) and GLYOXALASE I (GLOI) showed a higher accumulation at stage E3DO. In addition, a peak of transcript abundance in subordinate embryos was observed for transcripts related to WOX2, WOX8/9, AINTEGUMENTA-like 5 (PSAIL5), PHOSPHOGLUCAN WATER DIKINASE (PWD), MYOINOSITOL OXYGENASE 1 (MIOX1), ALFIN-like 3 (AL3) and a transcript encoding Auxin-dormancy-related protein.

ANACO09 is expressed in root cap stem cells where it promotes periclinal cell divisions [54]. FAMA, a basic helix-loop-helix protein, regulates a critical switch between division and differentiation during stomatal development [55]. In Norway spruce, the apical-basal polarization during early embryogeny proceeds through the establishment of the meristematic cells of the embryonal mass and the terminally differentiated, expanding suspensor cells [56]. The high expression of PsNACOO9 and PSFAMA at stage E3DO and E4, but not at stage E3SU, may reflect the importance of correct cell division pattern for the development of dominant embryos. PaWOX8/9 regulates the orientation of the cell division plane in the basal part of the embryonal mass during early and late embryogeny in Norway spruce [57]. In accordance, $P s W O X 8 / 9$ was expressed at all analysed developmental stages, however, the expression was significantly higher at stage E3SU. Although we do not know how overexpression of PaWOX8/ 9 affects early embryo development, it is tempting to assume that the high expression of PsWOX8/9 in subordinate embryos inhibits further development of the embryos or is only a consequence of a blocked development caused by other factors. In Arabidopsis plants overexpressing AtARPN, the endothecium degenerates, probably as a consequence of plantacyanin-induced precocious PCD [58]. The terminally differentiated suspensor cells in early embryos of Scots pine and Norway spruce are eliminated by PCD $[9,59]$. The high expression of PsARPN in E3DO and E4 embryos, but not in E3SU, coincides with the degeneration of the suspensor cells. Taken together, these results suggest that the apical-basal polarization is strictly regulated in dominant embryos but not in subordinate embryos.

Picea abies HOMEOBOX 1 (PaHB1), a homolog of AtPDF2, and PaWOX2 are important for specification of the protoderm in somatic embryos of Norway spruce $[60,61]$. Furthermore the expression of a Norway spruce LTP gene ( $\mathrm{Pa} 18$ ) switches from a uniform expression in proembryogenic masses to a protodermspecific localization in developing somatic embryos [62]. We assume that the differential expression of PSLTP4 and PSPDF2 is reflecting specification of the protoderm, which would indicate that radial patterning is regulated in a similar way in dominant and subordinate embryos. The expression pattern of PsWOX2 was similar to that of PsWOX8/9, both transcripts were expressed at all developmental stages, but with significantly higher levels in subordinate embryos. This is probably related to the blocked development of the subordinate embryos.

We have previously shown that $P S H A P 3 A$ is expressed during the morphogenic phase and PsVP1 during the maturation phase [63]. PsHAP3A was down-regulated in both E3DO and E3SU embryos, while PsVP1 was up-regulated in E3DO but not in E3SU embryos. 
Overexpression of the Arabidopsis EMBRYOMAKER (AtEMK), which is identical to AtAIL5, results in the formation of embryo-like structures on seedlings [64]. The authors concluded that $A t E M K$ is a key player to maintain embryonic identity. The PsAIL5 transcript was down-regulated in E3DO but not in E3SU embryos. Taken together, the low expression of PsVP1 and high expression of PsAIL5 in the subordinate embryos indicate that the transition from the morphogenic phase to the maturation phase is not completed in the subordinate embryos.

Although the functions of genes encoding for proteins related to PWD, MIOX1, AL3 and an Auxin-dormancy related protein during embryo development are not known, the fact that they are differentially expressed in E3DO and E3SU embryos indicates differences in metabolic processes between dominant and subordinate embryos.

Based on the processes identified in the GO enrichment analyses and the expression of the selected transcripts we suggest that processes related to embryogenic competence and cell wall loosening are involved in activating the cleavage process. Directly after cleavage, the growth of the embryos is restricted. Apical-basal polarization is strictly regulated in the dominant embryo, which has reached the maturation phase. However, functional studies must be performed before we will understand the processes controlling the successive development of the early embryos.

\section{Conclusion}

In this study we have analysed changes in transcript accumulation during early seed development. GO enrichment analysis of transcripts differentially expressed in embryos and megagametophytes highlighted the importance of different cellular processes and functions related to DNA replication and translation in embryos and accumulation of storage material and transcripts responding to different stimuli in megagametophytes. Transcripts related to embryogenic competence, cell wall modifications, cell division pattern, axis specification, and response to hormones and stress factors were highly abundant and differentially expressed during embryo development. The abundance of representative DETs during different stages of embryo development was verified by qRT-PCR analyses.

However, it has to be kept in mind that we have had to focus on genes that have been characterized in angiosperms. Further functional studies of the differentially expressed genes, and at least those which have been annotated to angiosperm genes without known function or that completely lack an angiosperm homolog, will increase our understanding of the regulation of embryo development in Pinus.

\section{Additional files}

\begin{abstract}
Additional file 1: Table S1. Number of 454 sequencing reads in the seed transcriptome. Table $\mathbf{S 2}$. Results from transcriptome assembly (trimmed by Seqclean). Table S3. Results from transcriptome integration. Table S4. Length of transcripts belonging to the seed transcriptome. Figure S1. Venn diagrams showing the number of common and unique transcripts and TFs with detectable expression $(\mathrm{RPKM}>0)$, at different developmental stages. Venn diagrams showing the number of transcripts at each developmental stage in (A) embryos and (B) megagametophytes. Venn diagrams showing the number of TFs at each developmental stage in (C) embryos and (D) megagametophytes. (PDF 393 kb)
\end{abstract}

Additional file 2: Table S5. List of transcripts selected for qRT-PCR analyses. Table S6. Validation of the RNA-seq expression data. (PDF $88.2 \mathrm{~kb}$ )

Additional file 3: Figure S2. Number of differentially expressed transcripts (DETs) with a fold-change greater than 2 ( $F C>2$ ) identified in each pairwise comparison between embryos and megagametophytes. (A) Total number of up-regulated transcripts in embryos and megagametophytes (B) Number of up-regulated transcripts in embryos (orange) and megagametophytes (green) at different developmental stages during early seed development. Figure S3. $\mathrm{GO}$ enrichment analysis of up-regulated transcripts $(F C>2$ ) in embryos, identified in any of the pairwise comparisons between embryos and megagametophytes at the four developmental stages shown in Fig. 1. Enrichment in Biological Processes was obtained using AgriGO Toolkit database (FDR <0.05). High significant levels are represented by red squares. Figure S4. GO enrichment analysis of up-regulated transcripts $(F C>2)$ in megagametophytes, identified in any of the pairwise comparisons between embryos and megagametophytes at the four developmental stages shown in Fig. 1. Biological Process enrichment was obtained using AgriGO Toolkit database (FDR <0.05). High significant levels are represented by red squares. (PDF $672 \mathrm{~kb}$ )

Additional file 4: Differentially expressed transcripts, with a fold change higher than 2, in at least one of the pairwise comparisons between embryos and megagametophytes. This file includes a list of all the upregulated transcripts in embryos and megagametophytes respectively, resulting from the pairwise comparisons E1 vs M1, E2 vs M2, E3DO vs M3, E3SU vs M3 and E4 vs M4. Transcript name (Transcript ID) and the best match against TAIR database (At locus ID, At name and At annotation) are shown. (XLSX 626 kb)

Additional file 5: Differentially expressed transcripts in embryos and megagametophytes at different stages of seed development. DETs with the highest fold-changes in embryos and megagametophytes at different developmental stages are included. Transcript name (Transcript ID), fold-change ( $\left.\log _{2} E / M\right)$, expression values (RPKM Embryos and RPKM Megagametophytes) and the best match against TAIR database (At locus ID, At name, At annotation) are shown. (XLSX $6.02 \mathrm{~kb}$ )

Additional file 6: Transcription factors (TFs) differentially expressed, with a fold-change higher that 2 , in any of the pairwise comparison between embryos and megagametophytes. A list of differentially expressed TFs (DETFs) in embryos and megagametophytes respectively, resulting from the pairwise comparisons E1 vs M1, E2 vs M2, E3DO vs M3, E3SU vs M3 and E4 vs M4 is included in this file. TF name (Transcript ID), TF family, and the best match against TAIR database (At locus ID, At name and At annotation) are indicated. The number of all DETFs detected for each family during seed development in embryos and megagametophytes is also included. (XLSX 200 kb)

Additional file 7: Figure S5. Distribution of up-regulated TF family members in embryos and megagametophytes. Presented data are based on TF family members differentially accumulated (FC $>2$ ) during seed development in any of the pairwise comparisons between embryos and megagametophytes. Orange bars show the number of TFs belonging to each family in embryos and green bars in megagametophytes. TFs were classified into TF families by using the publicly available PlantTFDB v 3.0 database. Figure S6. Abundance of the ten largest TF families differentially expressed between embryos and megagametophytes during seed development shown in Fig. 5. Number of members in each TF family detected at different developmental stages in (A) embryos and (B) 
megagametophytes. Subordinate embryos were excluded from this analysis. (PDF $312 \mathrm{~kb}$ )

Additional file 8: Figure S7. Number of differentially expressed transcripts (DETs) with a fold-change greater than 2 (FC > 2) identified in each pairwise comparison between embryo developmental stages. Upregulated and down-regulated transcripts are shown in red and green bars, respectively. The number of total DETs in each pairwise comparison is shown below. Table S7. Differentially expressed TFs (FC > 2), related to developmental process, detected in the pairwise comparisons between consecutive stages during embryo development. (PDF $341 \mathrm{~kb}$ )

Additional file 9: Differentially expressed transcripts ( $F C>2$; RPKM $>10$ ) between consecutive developmental stages during embryo development Only DETs with positive matches against TAIR database have been included. Transcript name (Transcript ID), fold-change (FC), expression values at each embryo developmental stage (RPKM) and the best match against TAIR database (At locus ID, At name, At annotation) are shown. GO enrichment analysis for the up- or down-regulated transcript list resulting from each pairwise comparison is also presented. For each $\mathrm{GO}$ enrichment result is shown the $\mathrm{GO}$ term, Onthology, $(\mathrm{P}=$ Biological Process, $\mathrm{F}=$ Molecular Function, $\mathrm{C}=$ Cell Component), Number of GO annotated transcripts belonging to each category among the up- or down-regulated transcript list (Number in input list), Number of total GO annotated transcripts belonging to each category in the seed transcriptome (Number in BG/Ref), $p$-value and level of enrichment (FDR). (XLSX $35.8 \mathrm{~kb})$

Additional file 10: Differentially expressed transcription factors ( $F C>2$; RPKM > 10), between consecutive developmental stages during embryo development. Up- and down-regulated TFs from each pairwise comparison, as well as the list and frequency of the TF families detected, are presented in this file. Transcript name (Transcript ID), fold-change (FC), expression values at each embryo developmental stage (RPKM), TF family and the best match against TAIR database (At locus ID, At name, At annotation) are indicated. TFs GO annotated for developmental biological process are coloured in red in the up-regulated TF lists and in green in the down-regulated TF list for each comparison. (XLSX $131 \mathrm{~kb}$ )

Additional file 11: Figure S8. Clustering of differentially expressed transcripts identified in the pairwise comparisons between different megagametophyte developmental stages. The analysis included DETs with a FC $>2$ and RPKM $>10$. For each transcript, RPKM values were normalized to its maximum RPKM value during development.

Normalized values were subjected to k-means clustering method and classified into different clusters, based on their expression levels across the four developmental stages. The $\mathrm{Y}$-axis of the cluster chart represents relative expression (from 0 to 1). The percentage of transcripts with $\mathrm{GO}$ terms assigned to different Biological Processes ( $\mathrm{GO}$ at level 2) for each type is shown. Figure S9. Differentially expressed transcripts between consecutive stages in megagametophytes during early seed development. The analysis included DETs, with FC $>2$ and RPKM $>10$, identified in any of the three pairwise comparisons. (A) Histogram showing the number of upand down-regulated DETs between different developmental stages. (B) Venn diagram showing the common and specific number of DETs detected in the consecutive pairwise analysis. (C) Summary table showing the number of DETs (excluding those with no assigned matches against TAIR) involved in different processes in each development transition. (PDF $1.23 \mathrm{~kb}$ )

Additional file 12: Differentially expressed transcripts ( $F C>2 ; R P K M>10)$ between consecutive megagametophyte developmental stages. Only DETs with positive matches against TAIR database are included. Transcript name (Transcript ID), fold-change (FC), expression values at each megagametophyte developmental stage (RPKM) and the best match against TAIR database (At locus ID, At name, At annotation) are shown. Transcripts GO annotated for developmental biological process are coloured in red or green, in the up- or down-regulated lists, respectively. All transcripts $G O$ annotated for response to stimulus and stress are highlighted in bold. (PDF $12.2 \mathrm{~kb}$ )

\section{Acknowledgements}

The authors would like to thank Gunnar Flygh (SLU) for the sample collection, Concepcion Avila and Pepi Gomez (UMA) for the RNA sequencing and Francisco Juan for his assistance with script building for data analyses.

\section{Funding}

This work was supported by the European Community's Seventh Framework Programmed under agreement No. 289841 (ProCoGen project).

\section{Availability of data and materials}

The sequence reads and expression data generated in this study has been deposited in The European Bioinformatics Institute (EBI) and are available in the ArrayExpress database (www.ebi.ac.uk/arrayexpress) under the accession number E-MTAB-5193. The RNA-seq data is also accessible through Gymno PLAZA 1.0 (http://bioinformatics.psb.ugent.be/plaza/versions/gymno-plaza/).

\section{Authors' contributions}

The original design of the study was set up by Sva and FC. MA contributed to tissue collection and gRT-PCR. BC isolated RNA and built the CDNA libraries for 454 pyrosequencing. LS did the transcript reconstruction from RNAseq. IM performed the bioinformatic analyses, RNA isolation, QRT-PCR, data analysis and manuscript organisation. IM and SvA reviewed the manuscript. All authors have read and approved the final manuscript.

\section{Competing interests}

The authors declare that they have no competing interests.

\section{Consent for publication}

Not applicable.

\section{Ethics approval and consent to participate}

Not applicable.

\section{Author details}

'Department of Plant Biology, Uppsala BioCenter, Swedish University of Agricultural Sciences, Box 708075007 Uppsala, Sweden. ${ }^{2}$ Department of Plant Systems Biology, VIB, B-9052 Ghent, Belgium. ${ }^{3}$ Department of Plant Biotechnology and Bioinformatics, Ghent University, B-9052 Ghent, Belgium. ${ }^{4}$ Bioinformatics Institute Ghent, Ghent University, Ghent B-9000, Belgium. ${ }^{5}$ Department of Molecular Biology and Biochemistry, School of Sciences, Campus de Teatinos, Universidad de Malaga, 29071 Malaga, Spain.

Received: 19 July 2016 Accepted: 28 October 2016

Published online: 18 November 2016

\section{References}

1. De Smet ILS, Mayer U, JLau S, Mayer U, Jürgens G. Embryogenesis - the Humble Beginning of plant life. Plant J. 2010;61(6):959-70.

2. Smith SA, Beaulieu JM, Donoghue MJ. An uncorrelated relaxed-clock analysis suggests an earlier origin for flowering plants. P Natl Acad Sci USA. 2010;107(13):5897-902.

3. Singh H. Embryology of Gymnosperms. Handbuch der Pflanzenanatomie. In: Zimmermann W CZ, Ozenda P, Wulff HD, editor. Gebrüder Borntrager, Berlin 1978. p. 187-241.

4. Chowdhury R. The embryology of conifers - a review. Phytomorphology. 1962;12:313-38.

5. Gifford E, Foster A. Morphology and Evolution of Vascular Plants. In: Co. WHF, editor. San Francisco: W.H. Freeman \& Co.; 1989. p. 440.

6. Buchholz J. Suspensor and early embryo of Pinus. Bot Gaz. 1918;66:185-228.

7. Buchholz J. Embryo development and polyembryony in relation to the phylogeny of conifers. Am J Bot. 1920;7(4):125-45.

8. Dogra PD. Seed sterility and disturbances in embryogeny in conifers with particular reference to seed testing and tree breeding in Pinaceae. Studia forestal suec Skogshogsk. 1967(45):97pp.

9. Filonova $\mathrm{LH}$, von Arnold S, Daniel G, Bozhkov PV. Programmed cell death eliminates all but one embryo in a polyembryonic plant seed. Cell Death Differ. 2002;9(10):1057-62.

10. Cairney J, Zheng L, Cowels A, Hsiao J, Zismann V, Liu J, et al. Expressed Sequence Tags from loblolly pine embryos reveal similarities with angiosperm embryogenesis. Plant Mol Biol. 2006;62(4-5):485-501.

11. Cairney J, Pullman GS. The cellular and molecular biology of conifer embryogenesis. New Phytol. 2007;176(3):511-36.

12. Vestman D, Larsson E, Uddenberg D, Cairney J, Clapham D, Sundberg E, et al. Important processes during differentiation and early development of somatic embryos of Norway spruce as revealed by changes in global gene expression. Tree Genet Genomes. 2011;7(2):347-62. 
13. Vega-Bartol JJd, Simoes M, Lorenz WW, Rodrigues AS, Alba R, Dean JFD et al. Transcriptomic analysis highlights epigenetic and transcriptional regulation during zygotic embryo development of Pinus pinaster. BMC Plant Biol. 2013;13(123).

14. Wang Z, Gerstein M, Snyder M. RNA-Seq: a revolutionary tool for transcriptomics. Nat Rev Genet. 2009;10(1):57-63.

15. Lu XD, Chen DJ, Shu DF, Zhang Z, Wang WX, Klukas C, et al. The Differential Transcription Network between Embryo and Endosperm in the Early Developing Maize Seed(1[C][W][OA]). Plant Physiol. 2013;162(1):440-55.

16. Jin FY, Hu LS, Yuan DJ, Xu J, Gao WH, He LR, et al. Comparative transcriptome analysis between somatic embryos (SEs) and zygotic embryos in cotton: evidence for stress response functions in SE development. Plant Biotechnol J. 2014;12(2):161-73.

17. Salvo SAGD, Hirsch CN, Buell CR, Kaeppler SM, Kaeppler HF. Whole Transcriptome Profiling of Maize during Early Somatic Embryogenesis Reveals Altered Expression of Stress Factors and Embryogenesis-Related Genes. Plos One. 2014;9(10).

18. Yang $X Y$, Zhang $X L$, Yuan DJ, Jin FY, Zhang YC, Xu J. Transcript profiling reveals complex auxin signalling pathway and transcription regulation involved in dedifferentiation and redifferentiation during somatic embryogenesis in cotton. BMC Plant Biol. 2012;12.

19. Zhang Y, Zhang SG, Han SY, Li XM, Qi LW. Transcriptome profiling and in silico analysis of somatic embryos in Japanese larch (Larix leptolepis). Plant Cell Rep. 2012;31(9):1637-57.

20. Canas RA, Canales J, Gomez-Maldonado J, Avila C, Canovas FM. Transcriptome analysis in maritime pine using laser capture microdissection and 454 pyrosequencing. Tree Physiol. 2014;34(11):1278-88.

21. Dong QF, Schlueter SD, Brendel V. PlantGDB, plant genome database and analysis tools. Nucleic Acids Res. 2004;32:D354-9.

22. Fu L, Niu B, Zhu Z, Wu S, Li W. CD-HIT: accelerated for clustering the nextgeneration sequencing data. Bioinformatics. 2012;28(23):3150-2.

23. Li H, Durbin R. Fast and accurate short read alignment with BurrowsWheeler transform. Bioinformatics. 2009;25(14):1754-60.

24. Li H. A statistical framework for SNP calling, mutation discovery, association mapping and population genetical parameter estimation from sequencing data. Bioinformatics. 2011;27(21):2987-93.

25. Jones P, Binns D, Chang HY, Fraser M, Li WZ, McAnulla C, et al. InterProScan 5: genome-scale protein function classification. Bioinformatics. 2014;30(9):1236-40.

26. Jin JP, Zhang H, Kong L, Gao G, Luo JC. PlantTFDB 3.0: a portal for the functional and evolutionary study of plant transcription factors. Nucleic Acids Res. 2014;42(D1):D1182-7.

27. Ye J, Fang L, Zheng HK, Zhang Y, Chen J, Zhang ZJ, et al. WEGO: a web tool for plotting GO annotations. Nucleic Acids Res. 2006;34:W293-7.

28. Du Z, Zhou X, Ling Y, Zhang ZH, Su Z. agriGO: a GO analysis toolkit for the agricultural community. Nucleic Acids Res. 2010;38:W64-70

29. Wickramasuriya AM, Dunwell JM. Global scale transcriptome analysis of Arabidopsis embryogenesis in vitro. BMC Genomics. 2015;16.

30. Yan DW, Duermeyer L, Leoveanu C, Nambara E. The Functions of the Endosperm During Seed Germination. Plant Cell Physiol. 2014;55(9):1521-33.

31. Li Q, Wang BC, Xu Y, Zhu YX. Systematic studies of $12 S$ seed storage protein accumulation and degradation patterns during Arabidopsis seed maturation and early seedling germination stages. J Biochem Mol Biol. 2007:40(3):373-81.

32. Tsuda K, Tsuji T, Hirose S, Yamazaki K. Three Arabidopsis MBF1 homologs with distinct expression profiles play roles as transcriptional co-activators. Plant Cell Physiol. 2004;45(2):225-31.

33. Teotia S, Muthuswamy S, Lamb RS. Radical-induced cell death1 and similar to RCD one1 and the stress-induced morphogenetic response. Plant Signal Behav. 2010;5(2):143-5.

34. Liu YQ, Jiang YS, Lan JB, Zou Y, Gao JP. Comparative Transcriptomic Analysis of the Response to Cold Acclimation in Eucalyptus dunnii. Plos One. 2014;9(11).

35. Friml J, Vieten A, Sauer M, Weijers D, Schwarz H, Hamann T, et al. Effluxdependent auxin gradients establish the apical-basal axis of Arabidopsis. Nature. 2003:426(6963):147-53.

36. Jenik PD, Gillmor CS, Lukowitz W. Embryonic patterning in Arabidopsis thaliana. Annu Rev Cell Dev Biol. 2007;23:207-36.

37. Larsson E, Sitbon F, Ljung K, von Arnold S. Inhibited polar auxin transport results in aberrant embryo development in Norway spruce. New Phytol. 2008;177(2):356-66.
38. Becerra C, Puigdomenech P, Vicient CM. Computational and experimental analysis identifies Arabidopsis genes specifically expressed during early seed development. BMC Genomics. 2006;7.

39. Carretero-Paulet L, Galstyan A, Roig-Villanova I, Martinez-Garcia JF, BilbaoCastro JR, Robertson DL. Genome-Wide Classification and Evolutionary Analysis of the bHLH Family of Transcription Factors in Arabidopsis, Poplar, Rice, Moss, and Algae. Plant Physiol. 2010;153(3):1398-412.

40. El Ouakfaoui S, Schnell J, Abdeen A, Colville A, Labbe H, Han S, et al. Control of somatic embryogenesis and embryo development by AP2 transcription factors. Plant Mol Biol. 2010;74(4-5):313-26.

41. Tang $W$, Ji $Q$, Huang $Y$, Jiang $Z$, Bao $M$, Wang $H$, et al. FAR-RED ELONGATED HYPOCOTYL3 and FAR-RED IMPAIRED RESPONSE1 transcription factors integrate light and abscisic acid signaling in Arabidopsis. Plant Physiol. 2013; 163(2):857-66.

42. Pascual BM, Canovas FM, Avila C. The NAC transcription factor family in maritime pine (Pinus Pinaster): molecular regulation of two genes involved in stress responses. BMC Plant Biol. 2015;15.

43. Li SZ, Zhou XJ, Huang YQ, Zhu LY, Zhang SJ, Zhao YF et al. Identification and characterization of the zinc-regulated transporters, iron-regulated transporter-like protein (ZIP) gene family in maize. BMC Plant Biol. 2013;13.

44. Wiweger $M$, Farbos I, Ingouff M, Lagercrantz U, Von Arnold S. Expression of Chia4-Pa chitinase genes during somatic and zygotic embryo development in Norway spruce (Picea abies): similarities and differences between gymnosperm and angiosperm class IV chitinases. J Exp Bot. 2003;54(393):2691-9.

45. Iglesias-Fernandez R, Barrero-Sicilia C, Carrillo-Barral N, Onate-Sanchez L, Carbonero P. Arabidopsis thaliana bZIP44: a transcription factor affecting seed germination and expression of the mannanase-encoding gene AtMAN7. Plant J. 2013;74(5):767-80.

46. Petrasek J, Friml J. Auxin transport routes in plant development. Development. 2009;136(16):2675-88.

47. Lewis DR, Ramirez MV, Miller ND, Vallabhaneni P, Ray WK, Helm RF, et al. Auxin and Ethylene Induce Flavonol Accumulation through Distinct Transcriptional Networks. Plant Physiol. 2011;156(1):144-64.

48. Chen F, Dahal P, Bradford KJ. Two tomato expansin genes show divergent expression and localization in embryos during seed development and germination. Plant Physiol. 2001;127(3):928-36.

49. Cosgrove DJ, Li LC, Cho HT, Hoffmann-Benning S, Moore RC, Blecker D. The growing world of expansins. Plant Cell Physiol. 2002;43(12):1436-44.

50. Hecht V, Vielle-Calzada JP, Hartog MV, Schmidt EDL, Boutilier K, Grossniklaus $U$, et al. The Arabidopsis SOMATIC EMBRYOGENESIS RECEPTOR KINASE 1 gene is expressed in developing ovules and embryos and enhances embryogenic competence in culture. Plant Physiol. 2001;127(3):803-16.

51. Yang WB, Gao MJ, Yin X, Liu JY, Xu YH, Zeng LJ, et al. Control of Rice Embryo Development, Shoot Apical Meristem Maintenance, and Grain Yield by a Novel Cytochrome P450. Mol Plant. 2013;6(6):1945-60.

52. Nakazawa M, Yabe N, Ichikawa T, Yamamoto $Y Y$, Yoshizumi T, Hasunuma K, et al. DFL1, an auxin-responsive GH3 gene homologue, negatively regulates shoot cell elongation and lateral root formation, and positively regulates the light response of hypocotyl length. Plant J. 2001;25(2):213-21.

53. Wu G, Gu Y, Li S, Yang Z. A genome-wide analysis of Arabidopsis Ropinteractive CRIB motif-containing proteins that act as Rop GTPase targets. Plant Cell. 2001;13(12):2841-56.

54. Willemsen $\mathrm{V}$, Bauch $\mathrm{M}$, Bennett $\mathrm{T}$, Campilho $\mathrm{A}$, Wolkenfelt $\mathrm{H}, \mathrm{Xu}$ J, et al. The NAC domain transcription factors FEZ and SOMBRERO control the orientation of cell division plane in Arabidopsis root stem cells. Dev Cell. 2008;15(6):913-22

55. Ohashi-Ito K, Bergmann DC. Arabidopsis FAMA controls the final proliferation/ differentiation switch during stomatal development. Plant Cell. 2006;18(10):2493-505.

56. Smertenko AP, Bozhkov PV, Filonova LH, von Arnold S, Hussey PJ. Re-organisation of the cytoskeleton during developmental programmed cell death in Picea abies embryos. Plant J. 2003;33(5):813-24.

57. Zhu TQ, Moschou PN, Alvarez JM, Sohlberg JJ, von Arnold S. WUSCHELRELATED HOMEOBOX 8/9 is important for proper embryo patterning in the gymnosperm Norway spruce. J Exp Bot. 2014;65(22):6543-52.

58. Dong J, Kim ST, Lord EM. Plantacyanin plays a role in reproduction in Arabidopsis. Plant Physiol. 2005;138(2):778-89.

59. Bozhkov PV, Filonova LH, Suarez MF, Helmersson A, Smertenko AP, Zhivotovsky $B$, et al. VEIDase is a principal caspase-like activity involved in plant programmed cell death and essential for embryonic pattern formation. Cell Death Differ. 2004;11(2):175-82. 
60. Zhu TQ, Moschou PN, Alvarez JM, Sohlberg JJ, von Arnold S. WUSCHELRELATED HOMEOBOX 2 is important for protoderm and suspensor development in the gymnosperm Norway spruce. BMC Plant Biol. 2016;16.

61. Ingouff M, Farbos I, Lagercrantz U, von Arnold S. PaHB1 is an evolutionary conserved HD-GL2 homeobox gene expressed in the protoderm during Norway spruce embryo development. Genesis. 2001;30(4):220-30.

62. Sabala I, Elfstrand M, Farbos I, Clapham D, von Arnold S. Tissue-specific expression of Pa18, a putative lipid transfer protein gene, during embryo development in Norway spruce (Picea abies). Plant Mol Biol. 2000;42(3):461-78.

63. Uddenberg D, Valladares S, Abrahamsson M, Sundstrom JF, Sundas-Larsson A, von Arnold S. Embryogenic potential and expression of embryogenesisrelated genes in conifers are affected by treatment with a histone deacetylase inhibitor. Planta. 2011;234(3):527-39.

64. Tsuwamoto R, Yokoi S, Takahata Y. Arabidopsis EMBRYOMAKER encoding an AP2 domain transcription factor plays a key role in developmental change from vegetative to embryonic phase. Plant Mol Biol. 2010;73(4-5):481-92.

\section{Submit your next manuscript to BioMed Central} and we will help you at every step:

- We accept pre-submission inquiries

- Our selector tool helps you to find the most relevant journal

- We provide round the clock customer support

- Convenient online submission

- Thorough peer review

- Inclusion in PubMed and all major indexing services

- Maximum visibility for your research

Submit your manuscript at www.biomedcentral.com/submit 